\title{
Design of "Smart Car Seat" to Prevent Heat Related Deaths of Children Left Alone inside Cars
}

\author{
Chao Li, Ph.D ${ }^{1}$, and Doreen Kobelo, Ph.D ${ }^{2}$ \\ ${ }^{1}$ Florida Agriculture and Mechanical University, USA, chao.li@famu.edu \\ ${ }^{2}$ Florida Agriculture and Mechanical University, USA, doreen.kobelo@ famu.edu
}

\begin{abstract}
Every year, there's news about children being left unattended by their guardians in the car and died from the heat related causes. The US government has realized the seriousness of the problem and started to pass some new regulations, demanding the car manufacturers to install some type of sensors inside the car to remind the adults about the kids being left in the car. The authors approach this problem from a different perspective. As we understand, not every car needs this type of sensors to detect kids because many cars don't transport children at all. But every child (year 5 and under) needs to sit on a car seat inside the car required by law. We are tackling the problem by adding more features to the car seat to make it "smart" enough to detect the temperature inside the car and to detect whether the kids are being left alone inside the car by the adults. The design utilizes a sensor platform and a smartphone application to reliably monitor the safety of the child. The system powers itself on in the presence of a child. The entire system is based on the programmable Arduino microcontroller circuit board. A force sensitive resistor is placed along the backrest of the car seat. This resistor is the indicator for the system to become active. When the system is active, meaning a child is present, the Arduino begins to monitor the temperature inside the car using a temperature sensor. When the temperature reaches unsafe level, an audible alarm is sent out. There is also a notification device paired with the system using app on the guardian's smart phone. Text messages will be sent to the phone if it is sensed that the parent is accidentally leaving the child behind in the vehicle. This part of the system is separate from the audible alarm that will sound if temperatures are reaching unsafe levels and will be strictly used for proximity. The double warning system will guarantee to alert the adult. The prototype has demonstrated the accomplishments of the functions and feasibility of the smart car seat in preventing the death of the kids.
\end{abstract}

Keywords-car seat, kids, heat related death, Arduino, sensor.

\section{INTRODUCTION}

Every year, there's news about children being left unattended by their guardians in the car and died from the heat related causes. According to [1], "the number of child hot car deaths for 2019 is currently 51 . On average, 39 children under the age of 15 die each year from heatstroke after being left in a vehicle. Nearly every state has experienced at least one death since 1998, and in 2018, a record number of 53 children died after being left in a hot vehicle."

Figure 1 demonstrates the dangers of an unattended vehicle that a child may have been left inside of. The temperature outside the car and inside of the car starts at $80^{\circ} \mathrm{F}$. The outside temperature remains the same throughout the entire hour and the interior of the car heats up 43 degrees, ending at $123^{\circ} \mathrm{F}$ [2]. According [3], even $80^{\circ} \mathrm{F}$ can be dangerous for a child under the age of one. These young children have not developed the ability to sweat and heat up five times faster than an adult. Figure 2 shows that two thirds of the heating inside of the vehicle take place in the first 30 minutes [4]. This means that after 30 minutes the temperature of the vehicle, in Figure 1, would already be around $108^{\circ} \mathrm{F}$. That temperature is fatal for a young child. This is the reason behind so many fatalities, because a child forgotten in the car has very little time to be remembered and saved. Often once remembered about, it is already too late. The results of a simple mistake are tragic. The study done by [5] focuses more on actual environmental conditions of cars heating up and provided much needed qualitative and quantitative data throughout the progression of the design process.

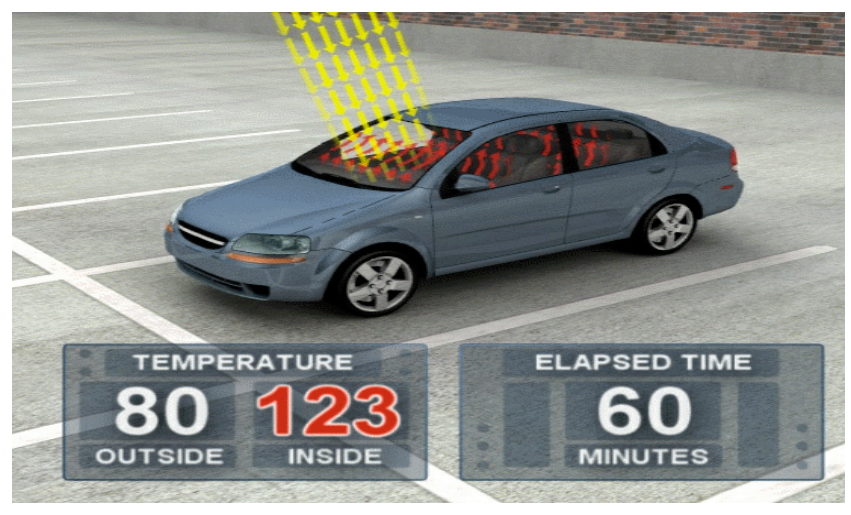

Fig. 1 Vehicle being Heated by Sun [2]

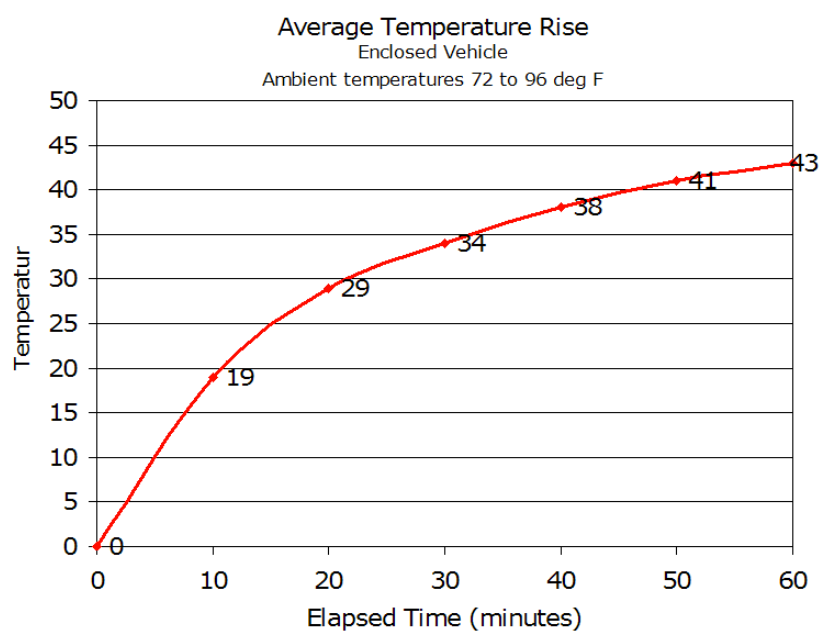

Fig. 2 Average Temperature Rise in an Enclosed Vehicle [4] 
The data collected from [5] is a much-needed source to demonstrate the seriousness of these fatalities across the United States. Until this study there was very limited conclusive data on this societal issue. The data collected proves that even though the numbers are not huge, there is a large enough trend where parents need a better system than what already exists to ensure the safety of their children's life. In [6], one researcher on this topic keeps detailed records of heatstroke deaths of children in vehicles. As of February 2020, there have been a total of 849 deaths that have been reported since 1998. 54 percent of these reported deaths are from parents forgetting about their child in the car, 25 percent are from children who were playing unattended in a vehicle, 19 percent are from parents that intentionally left children in a vehicle, and 1 percent is from unknown circumstances [6]. Over half of these deaths could have been prevented using the system proposed in this design and potentially more than that. This is because parents, who intentionally left their children in the car, may have decided against it had they been receiving temperature warnings from the phone application designed in this project.

Another key take-away from the study in [5] is that in the 10-year span that this study focused on, there were no child deaths in January and December. The major trend that this study displayed was that hyperthermia from children being left in cars increases, as the warmer seasons are present. This was to be expected, but the study gave the evidence needed to take from an assumption to a fact. This will result in the scope of the project relating to heat related hyperthermia incidents and not have any focus on cold weather incidents. However, that would be an excellent future rendition to the project. Figure 3 demonstrates the amount of deaths per month over the 10 -year span.

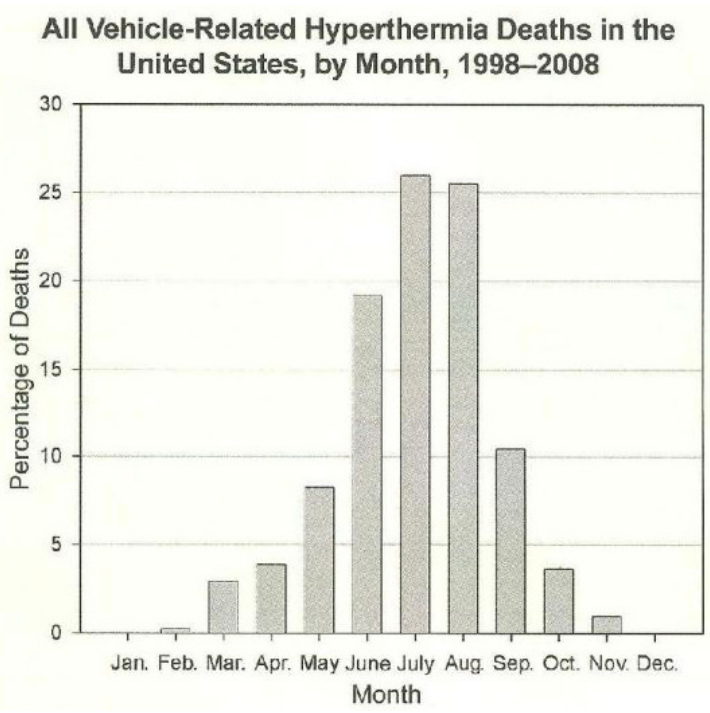

Fig. 3 Vehicle Related Hyperthermia Deaths [5]

A major skepticism people have in researching this topic is that there is no possible way that a parent could forget their child in the car, that the only parents who do must be terrible parents and that it could never happen to them. David Diamond, a neuroscientist from the University of South Florida, discussed his research on the brain and memory and how this tragedy could potentially happen to any parent or guardian. In [7], he claims that it is a competition in the brain between habit memory and prospective memory. "Prospective memory refers to the planning and execution of an action in the future, such as planning to take a child to daycare. Habit memory refers to tasks that involve repetitive actions that are performed automatically, as in routinely driving from one location to another, such as from home to work." He says that humans are a creature of habit and under stressful situations habit memory takes over and prospective memory is cast to the side. A new parent, who has not developed the habit of taking their child to daycare, is not unlikely to have habit memory take over, especially with a large stressor or sleep deprivation, and forget to bring child to daycare. This results in a child being forgotten in a vehicle. This research demonstrates societies dire need for a system that will notify of a child being forgotten in a car. Considering that a child can be forgotten based solely on human nature is not a comforting thought, and this design project aims to bridge that gap.

\section{PROJECT INTRODUCTION}

\section{A. Market Research}

There are some products available in the market to deal with this problem. However, for one reason or another, these products are not widely accepted and adopted by parent drivers.

Sense-a-Life (Fig 4) is a notification system that utilizes two major sensors. The first sensor monitors the child's presence and the second sensor monitors the guardian's presence. If the presence of a child and not a guardian is encountered, after a certain amount of time, the guardian's cell phone is notified. Sense-a-Life relies on two systems, both requiring separate batteries. There is potentially a higher risk of miscommunication with the more wireless sensors that are being utilized in this system. Also, the sensor underneath the driver's seat may signal false alarms if the driver is leaving the vehicle, but the passenger is to remain in the running vehicle while the driver is temporarily away.

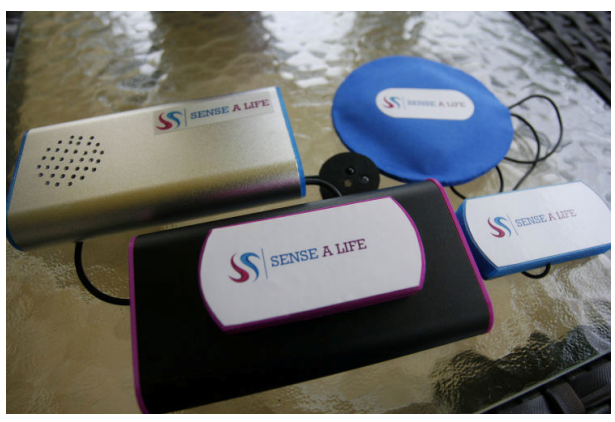

Fig. 4 Sense-a-Life System 
Drivers Little Helper (Fig 5) monitors interior temperature of vehicle, if driver gets out of seat, and the system will notify the driver whether he or she forgot the child in the car. The system does not have audible notifications. This system also encourages texting and driving, which leads to an entire new realm of ethical issues, especially with young children inside of the vehicle. The Smart Car Seat, on the other hand, has an audible notification system to allow the parent to know if temperatures are becoming unsafe while the vehicle is being driven. This is an ethical benefit because it encourages users to never use their cellphones while operating a motor vehicle.

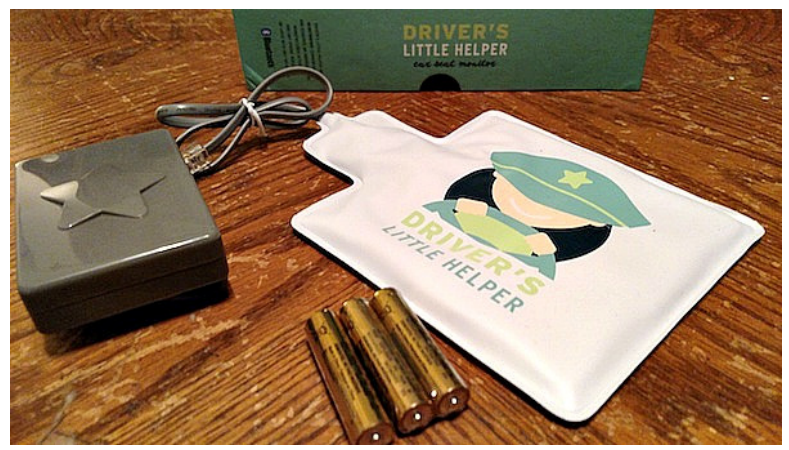

Fig. 5 Driver's Little Helper System

\section{B. Project Introduction}

This smart car seat project aims to create a reliable system with a very user-friendly interface. Since car seats are required for young children under the age of 5 in the US, embedded this system in the car seat is a natural choice. Research of other products on the market show that people are very interested in the safety concept, however products are currently not reliable and can be extremely frustrating. The frustration stems anywhere from connectivity issues to false alarms. The project design intends to bridge the gap of reliability paired with user friendliness.

The project aims to produce an easy to use smartphone application that seamlessly pairs via Bluetooth with the sensing component. The smartphone application is intended to display the current temperature of the child's immediate environment and notify the user if temperature conditions become unsafe. Another goal of the smartphone application is that if the user accidentally forgets the child inside of the vehicle they will be notified on their device.

The project scope also aims to utilize additional notification system that was not dependent on the functionality of a smart phone. This was the main difference between our design and what was currently available on the market. The idea behind this is that in the case of a Bluetooth malfunction, the user will still be notified of unsafe temperature conditions while their child is in the seat.

\section{METHODOLOGY}

Accurate temperature sensing played a vital role in the successful development of this project. Thermistors were considered as the mode of sensing temperature for this project. A thermistor is an electrical component that varies resistance based on a temperature input. But low-cost thermistors do not provide a precise enough measurement for the scope of the project. A TMP36 (Fig 6) is a low voltage, precision centigrade temperature sensor that provides an output voltage that is linearly proportional to the Celsius temperature with acceptable level of precision. The TMP36 was chosen to be the temperature sensor used in the design due to its very low cost and its precise temperature readings. Another reason the TMP36 was chosen over a thermistor is because they are more commonly used with the Arduino platform that was used in the design.

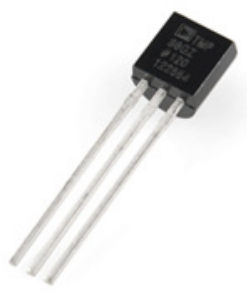

Fig 6 TMP36

A microcontroller was deemed necessary in the design process of the project. An idea that was considered was using a circuit that utilized logic gates to complete necessary tasks. The reason a microcontroller was deemed necessary was for future modifications. It is a more streamlined process to add in new features to the project through coding a microcontroller as opposed to designing a new logic circuit every time a change is necessary. Microcontrollers also enable system updates in the case that anything is not fully functional once the product hits the market. This could essentially prevent a product recall in the unfortunate event that something is wrong with the product. There is a plethora of available microcontroller kits on the market. The Arduino UNO was chosen because it is the market leader in open source hardware and has endless articles on troubleshooting issues encountered in nearly an endless number of different projects. The Arduino UNO had more than enough input and output pins, essentially making future modifications much more likely. The Arduino UNO does not require a large power supply to maintain functionality for extended periods of time, especially since the system being designed is not continuously powered on. The Arduino UNO was also an ideal choice for the system because it has a Bluetooth compatibility feature that can be readily integrated.

The JY-MCU(Fig 7) is Arduino's Bluetooth module. There are many compatible Bluetooth chips that can interface with the Arduino UNO, but the JY-MCU was chosen because it is recommended for use with the Arduino UNO. 


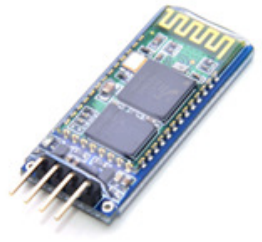

Fig 7: JY-MCU

A force sensitive resistor (FSR) in Fig 8 is a component that forms an open circuit until a force is applied to it. It is nearly a paper-thin component that fits in between the padding of the car seat and the car seat itself. When a child is placed in the car seat a force is applied to the circuit, the open is shorted and power is applied to the system. The main benefit of this is that the user does not have to remember to power the system on and off, instead the system powers on itself when it is needed. Another sensor considered to determine the child's presence was a leaf lever sensor. The sensor provides an open circuit until a force is applied and the leaf lever is suppressed resulting in a closed circuit. The reason that this sensor was ruled out in the design process was because it was bulkier than the force sensitive resistor and would be potentially uncomfortable for the child who was placed on top of the sensor.

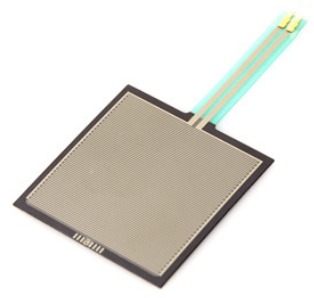

Fig 8: Force Sensitive Resistor (FSR)

A piezoelectric buzzer is an audio signaling device that was chosen to be used as an audible alarm built into the circuit. In case there is a malfunction between the phone notification system and the system, the system also has an audible alarm hardwired to sound if the system is on and temperatures become unsafe.

There is also a red and a yellow light emitting diode that is hardwired as a notification system. If the temperature warning sound is playing a yellow diode will power on and if the temperature is potentially fatal the red diode will power on.

In the beginning stages of design two of the biggest concerns were:

1) How will the system know if a child is present?

2) How will the user remember to turn on and off the system?
Switches and push buttons were two of the original ideas for powering the system $\mathrm{ON}$ and OFF. A major concern with this was that users could forget to turn on the system and have a false sense of confidence in their child's safety. It was decided that an ideal design would integrate the ability to turn $\mathrm{ON}$ and OFF on its own accord. This concept is what stemmed the idea of using a Force Sensitive Resistor (FSR). The FSR would be placed on the backrest of the car seat and wired in series with the power source. If no child is in the car seat there is no force applied to the FSR and the power input is an open circuit, but as soon as a child is placed into the car seat a force is present the power supply is no longer an open circuit and the system powers on. The FSR was the solution to two concerns encountered in the early stages of development. When tested in the prototyped circuit it was proven to be functional and did not pose any major issues on power input to the Arduino board. Figure 9 shows the hardware block diagram.

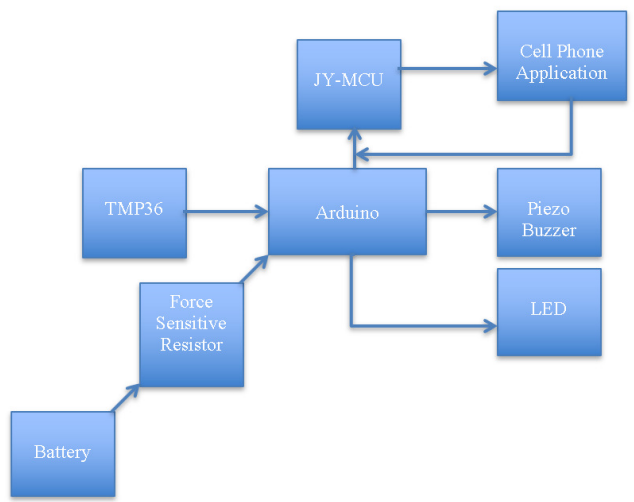

Fig 9: Hardware Block Diagram

MIT App Inventor 2 is open source application development software that enables users a streamlined process in creating applications for cellphones that can be uploaded directly to a device and tested [8]. In order to verify that the Bluetooth capabilities of the circuit were fully functional a prototyped application had to be created through MIT App Inventor 2. The application essentially provided basic means to connect from the device to the circuit and stream the incoming temperature data directly to the device. Prototyping the application helped to eliminate the learning curve required to fully develop the much more complex application delivered in the final product. Once the prototyped application was created, a temperature converting Arduino code was uploaded to the microcontroller. The test was successful, and accurate real time temperature readings were verified. The temperature was fluctuated using a heated fan, in order to test how quickly the readings responded to changes. The results proved to be nearly immediate.

A functionality issue was encountered with the Bluetooth after the initial design. It was realized that the MIT App Inventor 2 software was not able to detect an abrupt disconnection of Bluetooth. This means that if the handheld device gets out of range of the car seat the application will 
have no way of detecting this. The result is that it stores the last piece of data processed and continuously runs it through the code. A counter loop was attempted to be established, so that if the exact same piece of data were received an excessive amount of times with absolutely no fluctuation the application would recognize this to mean that Bluetooth had disconnected. In theory this seems like a feasible idea to monitor a parent accidentally leaving their child in the car. If the system is still considered to be on and they leave the range that Bluetooth is able to connect to, a red flag should be raised, and the user should be notified. This however is not the case, when Bluetooth is abruptly disconnected it is essentially a fatal error. Data stops being analyzed and enters an endless loop regardless of code set in place to attempt to catch this issue. This issue will need to be a future improvement once the MIT App Inventor 2 updates this issue with their software. In the meantime, this issue was evaded by re-evaluating methods that would work to monitor child safety. MIT App Inventor has a pedometer feature that utilizes the use of an accelerometer in the smartphone device, and after initializing the monitoring process, once ten steps are taken, regardless of if system is ON or OFF, a notification prompts the user to answer if their child was left in the car or if they are safe. A similar notification is prompted after twenty steps if there is no response in the first ten steps and again at thirty steps. This is a safety feature independent of the Bluetooth connection, so if for some reason Bluetooth connection is lost during the car ride, the user will still be notified to verify the safety of the child. Bluetooth is still utilized in the design to stream the temperature data from the car seat to user's device, but it is no longer a dependent variable to ensure safety of the child. This limitation resulted in a more efficient system, because it now essentially has a backup feature in case there are any errors in connectivity. Figure 10 shows the interface of the Android App developed.

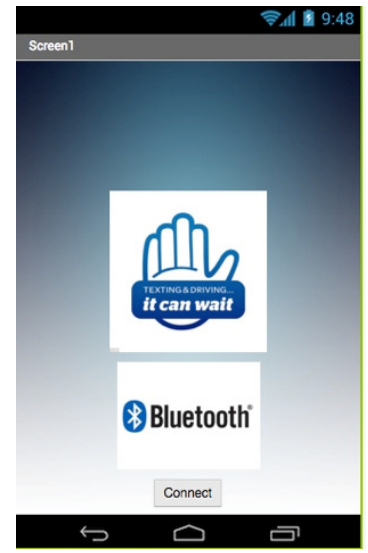

Fig. 10 Prototyped Application

The child's safety is the number one top priority of this project design. This goes beyond only monitoring the temperature and whether the child was left in the car. Users need to be able to add the system to a currently existing car seat without sacrificing the integrity of the car seat's crash test rating. The system should not pose a threat to the child inside of the car seat. There should be no choking hazards within reach and the battery pack must be far enough away from the child that in the unfortunate case of a malfunction the child's safety would not be jeopardized.

The battery pack and the circuit are essentially one component in the final design. This component is intended for the user to attach to the side of the car seat. This places a protective barrier in between the child and the system. In the case that a battery malfunctions, the child will not be directly exposed to the potentially life-threatening materials that a battery contains. The force sensitive resistor is the only piece of the system that extends out of the main system. A long wire that enables the user to place the force sensitive resistor in between the back pad of the car seat and the exterior cushion of a car seat. It is essential for child safety that the user is instructed to run the wire out of reach of the child and that the wire is long enough for any potential situation.

An added feature that will come with the system is a dangling toy that infants often have dangling over their car seats, the significance of the toy is that it has words written on it that the user will see every time they place their child in the car seat that reminds them to open the application and connect their device via Bluetooth before they begin driving.

\section{SOCIAL AND ENVIRONMENTAL IMPACT REPORT}

The design is intended to save the lives of children. Even new technology, with the best intentions, is subject to potential issues. The system contains a battery so the disposal of all the batteries will have an environmental impact. Users may become depended on the device for the safety of their child, when in reality it was designed to supplement safety. Further work on the project may raise concerns because the product intends to override all notification settings to alert the user that a child is in danger.

Eventually an upgraded version of the product will reach the market, or the batteries will expire and need to be replaced. In either of these cases many batteries will be disposed of. It is crucial that the consumer goes about proper battery disposal and does not simply throw the product away in the trashcan. Batteries contain hazardous waste and if enough people do not dispose of them properly, an influx of battery acid will be present at dumps resulting in negative effects on the environment. To combat this issue, a label will be placed on the battery, informing the consumer of the proper ways to dispose of the product, and the potential environmental hazards the battery poses if disposed of incorrectly.

The intentions of this design are not that parents will become fully reliant on ensuring child safety. The design is supposed to be a supplement that works alongside of good parenting skills. If users place all their trust into the product, some people may get the idea that the system can monitor their child who was left inside of the vehicle. Once temperature levels reach the warning level they return to cool 
down the child. This idea goes against everything that the design has tried to prevent. If users decide that the system is a safety net that enables them to leave their child in the car, then the product will need to be reevaluated in how to prevent this situation. The majority of users will most likely not abuse the system in this way, but the idea that there may be a small percentage who does is worrisome.

Finally, another social impact that future work on this project might raise are issues with privacy. The application intends to be able to override user notification preferences, so that every notification from the application is guaranteed to make noise. If the user has their phone on silent or vibrate the application eventually plans to be able to bypass this setting and issue a notification as loud as the speakers on the phone permits. The intentions are to have a higher chance of being heard and saving a child's life. The issue is that people do not like anything being able to change their personal settings. This feature could potentially raise privacy infringement concerns.

\section{CONCLUSION}

Leaving a child inside of an unattended vehicle is a serious situation no matter what the temperature outside of the vehicle is. Thankfully, advancements in technology provide society a crutch in their shortcomings. This project does just that, it takes away the possibility of forgetting a child in the car by constantly monitoring the safety of the aforementioned child and notifying the user of any unsafe findings.

A vehicle parked in direct sunlight heats up very quickly because there is a greenhouse effect where heat is trapped inside and has nowhere to escape. This is very dangerous for children to be left in at all. The younger the child, the less efficient their body is at cooling itself. Infants do not have the ability to sweat resulting in their internal temperature being prone to rise very quickly. Regardless of the age of the child, something must be done to prevent the forgetfulness of the human nature and this project aims solve this societal issue.

Products on the market already exist that attempt to do the same thing. What set this design apart from what already exists is the two separate monitoring systems incorporated into one design. Two separate parts of the system work side by side to monitor the same data. If temperature becomes unsafe the hardware components have a built-in audible and visible notification system to inform the user that temperatures are getting too hot. At the same time the Bluetooth component is working with the smartphone application to send a text message to the user if child is forgotten or if temperature becomes unsafe. These two parts do not rely on each other, so if the user for some reason does not have a Bluetooth connection, they will still be notified of unsafe temperatures.

Overall, a system was successfully developed that can monitor the wellbeing of a child inside of a vehicle. The system is capable of notifying the user if temperature conditions are becoming dangerous. A smartphone application was developed that can communicate with the system via
Bluetooth. An accelerometer inside of the smartphone was able to be utilized to monitor distance traveled by the parent while the system is active. All these advanced monitoring techniques are integrated into a very simple and very userfriendly device.

Market research proved that there is a need for this product and what is currently on the market is insufficient. This design made those much-needed improvements. Reliability was increased with a seamless Bluetooth connection. A backup feature was incorporated in the case that Bluetooth fails. The system is so reliable, that even if a user does forget that the system is present in their vehicle it will still monitor the child and notify in the case of unsafe temperatures.

\section{REFERENCES}

[1] https://injuryfacts.nsc.org/motor-vehicle/motor-vehicle-safetyissues/hotcars/

[2] Spector, Roseanne. "PARKED CARS GET DANGEROUSLY HOT, EVEN ON COOL DAYS, STANFORD STUDY FINDS." News Center. Stanford University, 05 July 2005. Web. 26 Feb. 2017

[3] Barth, Liza. "Dangers of Leaving Children in Hot Cars - Consumer Reports." Dangers of Leaving Children in Hot Cars. Consumer Reports, 23 May 2014. Web. 26 Feb. 2017.

[4] Null, Jan. "Fact Sheet - Heatstroke Deaths of Children in Vehicles." Fact Sheet - Heatstroke Deaths of Children in Vehicles. San Jose State University, 28 Feb. 2016. W eb. 02 Mar. 2017.

[5] Grundstein, Andrew, et al. "Weather, geography, and vehicle-related hyperthermia in children."The Geographical Review, vol. 101, no. 3, 2011, p. 353+. General

OneFile,go.galegroup.com/ps/i.do?p=ITOF\&sw=w\&u=tall $85761 \& \mathrm{v}=2.1$ \&id=GALE\%7CA268791332\&it=r\&asid=9a5f47c3ef3241bd28367ff213a cledb. Accessed 18 Mar. 2017

[6] http://www.noheatstroke.org

[7] Diamond, David. "Hot Car Deaths: How Can Parents Forget a Child in a Car?" CNN. Cable News Network, 25 July 2016. Web. 19 Mar. 2017.

[8] https://appinventor.mit.edu/ 